\title{
«Aspirare ad essere poeti di Lope»: las misceláneas in mortem como retrato del Madrid literario entre 1630 y 1640 \\ «Aspirare ad essere poeti di Lope»: The Miscellaneous in mortem as a Portrait of the Literary Madrid between 1630 and 1640
}

\section{Claudia Demattè}

https://orcid.org/0000-0002-9710-1689

Università di Trento

ITALIA

claudia.dematte@unitn.it

[Hipogrifo, (issn: 2328-1308), 9.1, 2021, pp. 143-157]

Recibido: 27-11-2020 / Aceptado: 08-01-2021

DOI: http://dx.doi.org/10.13035/H.2021.09.01.10

Resumen. El artículo analiza el fenómeno literario de los repertorios de funera dedicados a literatos españoles entre Italia y España, en particular la Fama póstuma a la vida y muerte del doctor frey Lope Félix de Vega Carpio (1636); las Essequie poetiche ovvero lamento delle muse italiane in morte del signor Lope de Vega publicadas en Venecia en 1636; y las Lágrimas panegíricas a la temprana muerte del gran poeta y teólogo insigne doctor Juan Pérez de Montalbán, clérigo presbítero y Notario de la Santa Inquisición, natural de la imperial villa de Madrid, lloradas y vertidas por los más ilustres ingenio de España (1639). El estudio subraya la participación tanto de famosos literatos como de jóvenes y ambiciosos poetas en estas misceláneas in mortem, además de sus relaciones con los mecenas del tiempo y con los libreros, en concreto con Alonso Pérez, en el contexto del Madrid entre 1630 y 1640. 
Palabras clave. Fama póstuma a la vida y muerte de Lope de Vega; Lágrimas panegíricas a la temprana muerte de Montalbán; Lope de Vega; Juan Pérez de Montalbán; Essequie poetiche ovvero lamento delle muse italiane in morte del signor Lope de Vega.

Abstract. The work analyses the literary phenomenon of the miscellaneous de funera devoted to spanish authors both in Italy and Spain between 1630 and 1640, especially the Fama póstuma a la vida y muerte del doctor frey Lope Félix de Vega Carpio (1636); the Essequie poetiche ovvero lamento delle muse italiane in morte del signor Lope de Vega published in Venice in 1636; and the Lágrimas panegíricas a la temprana muerte del gran poeta y teólogo insigne doctor Juan Pérez de Montalbán, clérigo presbítero y Notario de la Santa Inquisición, natural de la imperial villa de Madrid, lloradas y vertidas por los más ilustres ingenio de España (1639). The importance of the participation of famous authors but also young and ambitious poets in these works in mortem, will be investigated together with their relationships with patrons and booksellers in Madrid in the $17^{\text {th }}$ century.

Keywords. Fama póstuma; Lágrimas panegíricas, Lope de Vega; Juan Pérez de Montalbán; Essequie poetiche ovvero lamento delle muse italiane in morte del signor Lope de Vega.

No hay fuerza de paciencia que mitigue tanta ambición por el lugar primero. Lope de Vega, Elegía en la muerte de Villaizán

«Aspirare ad essere poeti di Lope» ${ }^{2}$ es el dictamen que todos y cada uno de los poetas que tratamos en nuestro trabajo siguieron por imitación o variación, durante toda su vida: el modelo que hay que conocer queda claro, «es [el] de Lope». Las palabras son del conde de la Roca, Juan Antonio de Vera y Zúñiga, embajador español en Venecia entre 1632 y $1642^{3}$, quien reconoce la indiscutible supremacía literaria de Lope en las Essequie poetiche ovvero lamento delle muse italiane in morte del signor Lope de Vega insigne ed incomparabile poeta spagnuolo publicadas en Venecia en 1636. El homenaje italiano llega un año después de la muerte del Fénix y posiblemente tras la publicación en Madrid de la Fama póstuma que salió a la venta en febrero de 16364. El texto de las Essequie poetiche se conoce principalmente por

1. Entrambasaguas y Peña, 1935, p. 144.

2. Ragguagli del Parnaso, en Essequie poetiche, p. 70.

3. Sobre su papel de embajador, ver López-Cordón Cortezo, 2015; Pineda, 2015 y Merluzzi, 2015. Nótese que solo López-Cordón toma en consideración Cinti (1966) mientras que ninguno de los dos demuestra conocer Cinti (1963) y Hempel (1964) ni citan las Essequie poetiche. Ver además Fernández-Daza Álvarez, 1994.

4. Aunque razonablemente la Fama póstuma preceda las Essequie, la escasa influencia del primer texto sobre el del conde de Roca nos lleva a dejar para la segunda parte de este trabajo el análisis de la Fama, un texto más conocido y estudiado, como veremos. Para las obras in mortem que se publicaron en la segunda mitad del siglo, ver Marcello (2013, pp. 955-956). Mientras este trabajo estaba en proceso de revisión, salió el estudio de Alejandro García-Reidy (2020) que recorre, junto a otros dos textos ocasio- 
su publicación en el volumen XXI de las Obras sueltas de Lope de Vega ${ }^{5}$ y declara ya en la portada que se trata de «rime e prose raccolte dal signor Fabio Franchi Perugino. Dedicate all'illustrissimo ed eccellentissimo signor don Gio. Antonio de Vera, e Figueroa Conte della Rocca» (p. 1). Como bien ha aclarado Bruna Cinti en sus estudios de los años sesenta, varios críticos se han dejado llevar por esta falsa pista atribuyendo al desconocido Fabio Franchi la obra, cuando la excesiva alabanza de la estirpe de los Vera invitaba a pensar que el verdadero autor podía ser el mismo Juan Antonio de Vera. Nicolás Antonio ya lo había insinuado y entre los modernos fue Dámaso Alonso el que volvió sobre el tema de la autoría ${ }^{6}$. Las investigaciones de Wido Hempel y una posterior monografía de Bruna Cinti dedicada al embajador aclararon no solo en qué medida Vera participó en la miscelánea sino también quién fue el erudito italiano que le ayudó: a pesar de que Hempel se inclinaba por el abad Alessio Pulci ${ }^{7}$, Cinti demostró que fue Agostino Rossi, traductor de otras obras del conde, dato que explica la falta total de hispanismos en el texto ${ }^{8}$. La obra consta de un prólogo, seguido por una Orazione fatta in Parnaso dal signor Cavaliere Marino in morte e lode del poeta d'Europa e Fenice Spagnuola, il signor Lope de Vega (pp. 13-20), a la que sigue un sinfín de poemas (pp. 21-60). La sensación es que uno se encuentra «continuamente en duda sobre la autenticidad de cada una de las composiciones» ${ }^{9}$. El núcleo de la obra se halla a continuación, se trata de Ragguaglio di Parnaso del sig. Fabio Franchi, alias conde de la Roca (pp. 61-73). Siguen numerosos poemas (pp. 73-160) y cierra la obra el Apostrofe nella morte del re de' poeti, Lope de Vega e Carpio del Molto R. D. Emanuel Tesauro (pp. 161-165). Las partes poéticas sobrepasan los cien poemas ${ }^{10}$, mientras que de los tres textos en prosa, el primero, la Orazione fatta in Parnaso, con toda seguridad se consideraría, entre los contemporáneos, tan solo como «una imaginaria y hábil ejercitación de retórica» ${ }^{11}$, ya que Marino había muerto diez años antes que Lope. Por otra parte, a propósito del Apostrofe, en un estudio reciente, Marco Maggi confirma la autoría, puesta en duda en su momento por Cinti, gracias a numerosas analogías con el estilo de Emanuele Tesauro ${ }^{12}$. El texto que, sin embargo, considero central para nuestro tema es el Ragguaglio di Parnaso, ya que retrata la audiencia que piden algunos poetas españoles a Apolo al día siguiente de las exequias de Lope de Vega en el templo délfico. Apolo se hace acompañar de Homero, Séneca, Tasso, Sannazaro y Anibalcaro, mientras Lope de Rueda habla por el grupo de unos quince «poeti comici» (p. 62) que le acompañan, todos ellos antepasados del

nados por la muerte de Calderón y de sor Juana Inés, la Fama póstuma, las Essequie y las Lágrimas que abarcamos también en nuestro trabajo, aunque fijándose en aspectos diferentes de estas extensas misceláneas.

5. Vega y Carpio, Obras sueltas, pp. 1-165. Las citas proceden de esta edición y las páginas se indicarán entre paréntesis en el texto.

6. Para un repaso de la cuestión de la atribución a lo largo del siglo xx, ver Cinti, 1963, pp. 609-611.

7. Hempel, 1964, pp. 39-45.

8. Cinti, 1963, p. 620 y Cinti, 1966, pp. 35-37 y especialmente la nota 95.

9. Cinti, 1963, p. 618.

10. Maggi, 2003, p. 428

11. Cinti, 1963, p. 616.

12. Maggi en su estudio edita en apéndice el Apostrofe con copiosas anotaciones (2003, pp. 428-437). 
«Secol d'oro» (p. 62): Montemayor y Torres Naharro, entre ellos. Los poetas acuden para pedir a Apolo dos cosas: en primer lugar, que se quemen todas las obras que ellos mismos publicaron hasta hace cuarenta años y que las sucesivas se expurguen $^{13}$. En segundo lugar, ya que Apolo ha otorgado a la excelsa España

un esemplare ed una tramontana sicura per non fallire (questo è il GRAN LOPE) ti degni [...] di comandare con decreto irrefragabile, che tutti li poeti lirici imitino in quanto sarà loro possibile (già che impossibile sarà in tutto) il numero, tenerezza e concetti delle rime del Fenice Lope (p. 65).

Antes de analizar las razones que alega Lope de Rueda para semejante petición, hay que observar que un decenio antes otro escritor italiano, Scipione Errico, había puesto en escena un tribunal similar. En su comedia Le rivolte di Parnaso (1625) las más altas autoridades de las letras italianas, Ariosto, Tasso, Trissino, Marini, se presentan delante de Apolo, pero de repente se ven interrumpidos por:

Lope di Vega con una moltitudine di Spagnoli a perturbare il cervello, domandando che le Tragedie e Comedie loro fossero degne dell'immortalità, ancorché non fosse conforme li precetti d'Aristotele e d'altre leggi poetiche, che l'altre nazioni osservano: e mi chiesero licenza che il tempo dell'azioni, in vece dello spazio d'un giorno, possa essere il termine di trecento o quattrocent'anni; la scena non fosse in loco determinato, ma in tutto il mondo, e nel medesimo tempo fosse or camera secreta, or pubblica loggia ${ }^{14}$

Apolo pide consultarlo con las Musas pero el alboroto de los españoles le obliga a aceptar la petición y a alejarlos por la fuerza. Posiblemente Juan Antonio de Vera no hubiese quedado satisfecho de la fama de prevaricadores que el escritor siciliano les había atribuido a los españoles, y sobre todo a Lope de Vega, y consideró que las Essequie serían una excelente ocasión para vengarse literalmente al describir de forma puntual las calidades de cada uno de los dramaturgos que siguió «lo spirito e il manto del loro maestro Lope» (p. 66). Se trata de una quincena de nombres, entre los cuales se encuentran Calderón, Tirso, Amescua, Coello y Alarcón (pp. 66-69). Como botón de muestra del conocimiento que tiene el conde de la Roca del teatro contemporáneo, citamos la primera alusión que es a Juan Pérez de Montalbán. El anhelo es que

scrivano e continuino nel far le Commedie, senza che al felice (fin ora) Montalbano le dia fastidio la censura de quel critico che nella sua commedia della Biscaina ${ }^{15}$ disse che fidò molto dell'auditorio, pretendendo, che un viso medesimo ingannasse in tre corpi diversi, che scriva sempre, e scriverà come si conviene, a tanto natural dotto, ed erudito Poeta, che riconoscendo nell'imitazione per suo padre il gran Dedalo Lope, resterà sicuro di non cascare come Icaro (p. 66).

13. «... che mandiate ad abbruciare tutte le nostre opere che sono fatte da quaranta anni addietro e quelle di quaranta anni in qua le mandiate a purgare» (p. 63).

14. Errico, 1974, pp. 83-84; Tedesco, 2006, pp. 231-232, Conrieri y Vuelta García, 2011, y Vuelta García, 2012.

15. Se trata de La toquera vizcaína (Pérez de Montalbán, Primer tomo de comedias, vol. 1.4, pp. 155-310). 
De cada dramaturgo se cita alguna comedia y por ejemplo, en el caso de Antonio de Solís y Francisco de Rojas se ordena que cada uno escriba por lo menos doce comedias cada año, «poiché quelle che hanno fatto non hanno avuto altro difetto ch'esser poche» (p. 68). De repente el discurso de Lope de Rueda se ve interrumpido por la llegada de Jerónimo de Villaizán, quien interviene para dejar sentada su crítica en contra de las comedias en colaboración. Hay que recordar que Villaizán había muerto prematuramente, poco antes de cumplir los treinta años, en 1633 cuando se hallaba entre los autores preferidos por el rey, como él mismo confirma a Apolo «avendo fatto la commedia che intitolai De un agravio tres venganzas che meritò l'udienza del Giove ed Apollo della sfera terrestre Filippo IV» (p. 71). Su súplica es para que Apolo prohiba

la unione o lega che sogliono far tra di loro, tre o quattro poeti [...] perché se questo stile s'introduce e radica, si vedranno mostri e non commedie. [...] più somiglia [...] una congiura che una commedia perché in tre atti vedersi tre stili differenti, altro non è che convertire il teatro in deserto di Libia dove si vedono Mostri di varie specie (p. 71).

Villaizán, al que le habían "forzado" a participar en «una commedia comune di tre nella quale perdessimo non solo l'olio e l'opera ma anche il credito per prima acquistato» (p. 71), afirma que todas las comedias en colaboración tendrían que prohibirse a excepción de Los tres blasones «fatta per tre grandi ingegni, che anco la stimo per mostro di beltà come tutte le altre per mostro di bruttezza» (p. 72) ${ }^{16}$. Escuchadas las palabras de Villaizán, y después de una breve consulta con Tasso y Lucano, Apolo decreta que Mercurio viaje a España para notificar a los poetas nombrados que juren «di difendere ed osservare ed aver per esemplare per far le loro commedie ed anco ogni componimento lirico le regole e stile del grande, insigne ed incomparabile Lope de Vega» (p. 72). La declaración de estima de Juan Antonio de Vera hacia el Fénix quedaba pues bien clara para todo el público italiano que tenía en sus manos un breve pero eficaz compendio del arte dramático contemporáneo español y también noticia, si bien crítica, de una nueva moda, la de las comedias de consuno, que estaba arraigando en la década de 163017. La intervención de Villaizán en la obrita del Conde de la Roca recuerda a los lectores que este autor había muerto pocos años antes: el mismo Lope de Vega le había dedicado una Elegía18, como había hecho por otra parte pocos meses antes en ocasión de la muerte de su querido amigo Paravicino ${ }^{19}$. Estas composiciones in mortem quedan fuera de nuestro trabajo por haber aparecido singularmente y no a raíz de un plan editorial de una obra de conjunto, una obra miscelánea tal y como la pensó Juan Pérez de

16. Se trata, en realidad, de una comedia en colaboración entre dos ingenios: Coello la primera jornada y Rojas las otras dos (Rojas Zorrilla, Obras completas. Volumen VI: Segunda parte de comedias).

17. El fenómeno de la comedia en colaboración ha sido últimamente bastante estudiado, ver Alviti, 2017 para una bibliografía actualizada sobre el tema. Como se sabe, Lope frecuentó bien poco este género (Demattè, 2017).

18. Para la Elegía en la muerte de Villaizán, ver Entrambasaguas y Peña, 1935 y Dixon, 1961.

19. La elegía en la muerte de Fray Hortensio Paravicino, titulada «Eliso» se publicaría póstuma en La Vega del Parnaso. 
Montalbán cuando murió Lope de Vega en el agosto de 163520. La Fama póstuma a la vida y muerte del doctor frey Lope Félix de Vega Carpio y elogios panegíricos a la inmortalidad de su nombre, como bien destaca Enrico Di Pastena, era un libro que «se inscribía en la floración barroca de los repertorios de funera, con la peculiaridad de estar consagrado a un literato» ${ }^{21}$. Remito al detallado estudio de Di Pastena en su edición crítica para el análisis del texto, pero aquí quiero subrayar las dudas que se plantearon a lo largo de los siglos acerca de la participación del duque de Sessa en la selección de las aportaciones y el papel de Montalbán en la obra. La presencia del escritor madrileño en la obra, con nueve poemas, la biografía de Lope y el prólogo, nos permite sospechar que todo su afán de restarse importancia, y atribuírsela al duque de Sessa, a la hora de tratar de la responsabilidad en decidir los participantes y organizar la estructura del volumen, respondiese más bien a un topos literario. No cabe ninguna duda que no solo por su afecto a Lope sino también por ser hijo del librero Alonso Pérez, se encargó de «solicitar a los que escriben, recoger sus versos, ponerlos en un tomo, presentarlos en el Consejo Real, llevarlos a los jueces, sacar licencia para imprimirlos, rubricarlos del escribano de cámara y trasladarlos del papel al bronce, sin más interés que mostrar al mundo el amor que siempre tuve a este gran varón» ${ }^{22}$. Sea como fuese, la Fama póstuma consta de ciento setenta y siete composiciones poéticas, una comedia de Gabriel de Moncada, Honras a Lope de Vega en el Parnaso, y seis textos en prosa: la primera biografía de Lope y el prólogo, como textos liminares, los dos a cargo de Montalbán; la oración fúnebre de Godínez, el discurso evangélico y moral de Sebastián de Medrano, la Urna sacra de José Pellicer ${ }^{23}$ y la carta de Juan de Solís. Publicada en 1636 a costa de Alonso Pérez, la Fama póstuma había obtenido el privilegio en diciembre de 1635 y no tuvo reimpresiones, si pasamos por alto que en 1779 se incluyó en el volumen XX de la Colección de las obras sueltas, así en prosa como en verso, de don Frey Lope Félix de Vega Carpio del editor madrileño Antonio de Sancha ${ }^{24}$. Si los funerales del Fénix fueron dignos de un miembro de la familia real por participación y extensión, la publicación que reunió Juan Pérez de Montalbán fue digno de un rey, tal y como fue Lope, para el mundo de las letras. Sin embargo, la miscelánea tuvo una eco efímera, dato que refleja cómo pronto Lope de Vega dejó de ser una marca comercial, dejando el paso a otros grandes, como Pedro Calderón de la Barca ${ }^{25}$. No me detengo más en esta obra que ya ha merecido la atención de varios estudiosos ${ }^{26}$, para pasar a la que se puede considerar, en cierta medida, una imitación de la Fama póstuma

20. Para un panorama de las obras que se escribieron a raíz de la muerte del Fénix, ver Blanco, 2014. Por otra parte, no era la primera vez que Montalbán se dedicaba a una obra miscelánea ya que en 1632 había publicado el Para todos (Demattè, 2003; Pérez de Montalbán, 1999).

21. Di Pastena, 2001, p. XIV.

22. Pérez de Montalbán, Fama póstuma, p. 14.

23. Sobre la participación de Pellicer en la Fama póstuma y un epitafio anterior, Burguillo, 2019.

24. Pérez de Montalbán, Fama póstuma, pp. CII-CXI. Ver Zimic, 1975 y Sánchez Jiménez, 2011.

25. A este propósito ver Vega García-Luengos, 2013 y Trambaioli, 2016.

26. Remito a los estudios de Di Pastena, 2001; Blanco, 2014; Trambaioli, 2016 y García-Reidy, 2020, y a la bibliografía allí recogida. 
y que surge de la muerte de Juan Pérez de Montalbán, a los 36 años, acaecida el 25 de junio de 1638 después de una enfermedad que sufrió a partir de la muerte de Lope y que lo redujo al estado de niño en un asilo de Madrid²7.

Las Lágrimas panegíricas a la temprana muerte del gran poeta y teólogo insigne doctor Juan Pérez de Montalbán, clérigo presbítero y Notario de la Santa Inquisición, natural de la imperial villa de Madrid, lloradas y vertidas por los más ilustres ingenios de España se publicaron en 1639 gracias a «la estudiosa diligencia del licenciado don Pedro Grande de Tena, su más aficionado amigo» y se ofrecieron al padre del difunto, el famoso librero madrileño Alonso Pérez. El volumen se compone de 11 folios sin numerar de paratextos y 164 folios numerados de poesías y textos en prosa, como por ejemplo la Idea de la comedia de Pellicer (fols. 146r$152 \mathrm{v})^{28}$. Al volumen se añaden, con numeración aparte, pero en diferente orden en los distintos ejemplares que se conservan de la primera edición 29: la Poesía defendida de Marqués de Careaga (fols. 1r-18r), el Elogio evangélico funeral de Diego de Niseno (fols. 1-34) y la Oración panegírica de Quintana ${ }^{30}$ (fols. 1r-14v). Mientras que la Poesía defendida tiene tan solo numeración independiente, pero carece de paratexto o portada con datos tipográficos, los otros dos se presentan como publicaciones independientes y en un segundo momento, posiblemente, insertadas en las Lágrimas. El Elogio evangélico funeral de Diego de Niseno presenta la aprobación del abad del Monasterio del Gran Basilio de Madrid, Diego Pinedo, con fecha 14 de junio de 1639, mientras que la aprobación de Juan Baptista Dávila puesta al final de la Oración panegírica es del 20 de junio de 1639. Ha pasado un año exacto de la muerte de Montalbán y el volumen de las Lágrimas está listo para la conmemoración ${ }^{31}$.

27. Para unas noticias más detalladas sobre los últimos años de la vida de Montalbán, ver Dixon, 2013, pp. 532-533 y Demattè, 2019, pp. 3-11.

28. Profeti, 1966-67.

29. A este propósito nótese que, en la Suma de la Tasa de las Lágrimas, Miguel Fernández afirma que el volumen se tasa a «cuatro maravedís y medio cada pliego, el cual tiene cuarenta y cuatro pliegos, sin los Sermones» (f. [6r]), es decir, 11 folios de paratexto y 164 de texto, dato que nos confirma que la intención de Pedro Grande de Tena era un volumen que incluyese la Oración y el Elogio. El ejemplar de la Biblioteca de Catalunya que se presenta completo de los 164 folios de las Lágrimas, seguidos con numeración distinta de La poesía defendida de Marqués de Careaga (fols. 1-18); la Oración panegírica de Quintana y el Elogio evangélico funeral de Niseno (fols. 1-34v). La misma estructura consta en el ejemplar de la Biblioteca de Granada (disponible en línea) y en el ejemplar de la Biblioteca Nacional de España R30820 (en Biblioteca Digital Hispánica). El ejemplar de la BNE (R199497) presenta la Poesía defendida (sin algún paratexto ni aprobación) antes del prólogo «Al lector y a quien le oyere de Lorenzo de Urnieta y Aguirre» al que siguen las Lágrimas, para acabar con el Elogio evangélico funeral y la Oración panegírica con sus respetivos paratextos. El ejemplar de la Österreichische NationalBibliothek presenta los fols. 1-164 seguidos por la Oración fúnebre mientras que le falta el Elogio Evangélico Funeral de Niseno (disponible en línea). Citamos de ahora en adelante del ejemplar de la Biblioteca de Catalunya, con signatura R(8)-8-329, entre paréntesis en el texto.

30. Bresadola, 2012.

31. Después de las aprobaciones de Diego de Niseno y de Bautista Dávila otorgadas en febrero, la Suma de Privilegio se había concedido en marzo del mismo año, mientras que tenemos que esperar hasta septiembre para que Murcia de la Llana firme la Fee de Erratas. 
Las Lágrimas se presenta como un conjunto de homenajes in mortem de 186 entre autores y autoras que pertenecen a distintos ambientes sociales: hay cierto número de personajes extraños al círculo de las letras, es decir, miembros de la sociedad del tiempo; hay un grupo bastante significativo de dramaturgos y otro de autoras, entre las cuales podemos distinguir a las escritoras profesionales como María de Zayas (51v) y Bernarda de la Cerda $(134 \mathrm{v})^{32}$, a las religiosas de diferentes monasterios de Madrid, Valladolid, Sevilla, Salamanca y Segovia y a un restringido número de damas de las que todavía desconocemos sus relaciones con el difunto ${ }^{33}$.

En palabras de Victor Dixon, y comparando la Fama póstuma con las Lágrimas, «it might seem surprising today that his young disciple appears to have been no less admired and loved» ${ }^{34}$ : a pesar de que cínicamente podríamos pensar que ningún otro escritor podía contar con un padre que costease una publicación similar, no hay que dejar a un lado la oportunidad que muchos aprovecharon para vengarse de los envidiosos, un tema que desasosegó durante mucho tiempo al mismo Montalbán (piénsese en la polémica surgida a raíz de la Perinola de Quevedo) ${ }^{35}$. En realidad, muchos de los participantes guardaban con Montalbán una sincera relación duradera, como por ejemplo de Tirso de Molina ${ }^{36}$, y casi todos frecuentaban la tienda de su padre, un lugar que se confirma como un centro de relaciones literarias y políticas muy importante ${ }^{37}$. Pero las Lágrimas no fueron el único testigo de la amplia estima de la que gozaba el «Fénix de la Europa»38, utilizando las palabras de la silva de González de Varela, sino que salieron otras contribuciones, al mismo tiempo o algún tiempo después, como destacara Victor Dixon ${ }^{39}$. En primer lugar, sobresale el breve tratado titulado La poesía defendida de Marqués de Careaga, escrito el 26 de junio del año 1639, es decir un año exacto después del fallecimiento de Montalbán y que acabó incorporado en las Lágrimas. En varios ejemplares este tratadito de 18 folios con numeración aparte se halla inmediatamente después de los 164 folios de las Lágrimas; sin embargo, el ejemplar conservado en la Biblioteca Histórica de la Universidad Complutense (BH FLL 37231) que carece de portada, presenta una encuadernación peculiar porque presenta el tratado de Marqués de Careaga como primer texto. En La poesía defendida se procede en 17 apartados y se deja tan solo para el final la referencia a nuestro Montalbán, como si fuese un alegato posterior para justificar la inserción de este texto en las Lágrimas al recordar su éxito en las tablas madrileñas «cosa que jamás se ha visto ni dicho de

\section{Baranda, 2003, pp. 237-238}

33. Ver Profeti, 2005, Baranda, 2007 y Martos Pérez, 2017. Lola Luna tan solo enumera en orden alfabético las escritoras presentes sacándolas del índice y sin añadir ninguna información más «cuyo índice ofrece 19 nombres femeninos» (1996, p. 74).

34. Dixon, 2013, p. 532

35. A propósito de las acusaciones de Quevedo a Montalbán, acerca de su supuesto judaísmo y de su dependencia de Lope, y de las defensas que levantaron varios literatos, ver Del Piero, 1961 y Plata, 2006. 36. Sobre la amistad entre Tirso y Montalbán ver los dos estudios de Ruth Lee Kennedy (1972-1973 y 1973-1974).

37. Cayuela, 2005, p. 139. Ver también Dadson, 2011, p. 26: «Para el amante de la poesía, la librería madrileña de Alonso Pérez tenía que haber sido el paraíso en la tierra».

38. Lágrimas, fol. 155r, del licenciado Joseph González de Varela (silva de heptasílabos y endecasílabos). 39. Dixon, 2013, p. 533. 
ninguno, aunque entre en ellos el Fénix deste arte y el Fénix de su amistad, LOPE» ${ }^{40}$. Claro que la primacía sobre Lope razonablemente la interpretamos en línea con la alabanza a Montalbán en una miscelánea de funera dirigida al padre del difunto.

En segundo lugar, tomamos en consideración tres composiciones que recuerdan la muerte todavía reciente del escritor madrileño: una décima de Francisco de Lyra y dos décimas y un romance de 40 versos de Brígida de Orduña se incluyen en la edición de 1641 de los Sucesos y prodigios de amor publicada en Sevilla por Nicolás Rodríguez. Nótese que esta edición reproduce los paratextos de la primera edición de 1624, pero añade los poemas citados y una licencia extendida a «Juan López Román para imprimir estas novelas» de noviembre de 1641. Este librero sevillano comerciaba libros en el Nuevo Mundo ${ }^{41}$ y, según Cayuela ${ }^{42}$, mantenía contactos con Alonso Pérez, quizás a partir del gran éxito que tuvo la colección de novelas de su hijo en México ${ }^{43}$. Si sobre la identidad de doña Brígida, monja en Santa Paula de Sevilla, quien había participado también en las Lágrimas con una canción (fol. 55r), no hemos podido recopilar más informaciones, sabemos por sus versos que la estima hacia Montalbán como autor era desde luego muy alta: lo define «solo / único Fénix de Lope» (fol. 5r) y «raro prodigio del Orbe» (fol. 5v). Por otra parte, nos llama la atención la participación del que podemos considerar uno de los impresores más importante en Sevilla en los años 1615-1645, Francisco de Lyra $^{44}$, con una décima que podríamos aventurar que derive quizás de los contactos comerciales con el padre del difunto.

Cierra esta pequeña galería de homenajes in mortem, destacando por darse casi quince años después de la muerte, un soneto en las Rimas (1652) de Juan de Moncayo y Gurrea, marqués de San Felices, «A la muerte de Juan Pérez de Montalbán, ingenio felicísimo de España, malogrado en lo mejor de su edad con la pérdida del juicio» ${ }^{45}$, que está precedido por un soneto a la muerte de Paravicino («Moriste dando el bronce de tu fama», p. 30) y otro a la de Lope («Moriste y el peso de tus años», p. 31), los únicos de los Sonetos fúnebres que se dedican a literatos del tiempo entre varios dedicados a personajes políticos y a una «dama muerta» (p. 35).

Volviendo a las obras misceláneas in mortem, parece interesante observar cómo los participantes confirman una vez más que los autores que escribían en estas obras colectivas eran principalmente los mismos ${ }^{46}$. Osuna subraya que «el elenco de autores sugiere un entorno cortesano relativamente reconocible, pese a las notables diferencias de edad e incluso de orientaciones estéticas»: en primer lugar están presentes varios dramaturgos: Calderón de la Barca, Luis Vélez de

40. Lágrimas, fols. 17v-18r.

41. Rueda Ramírez, 2012

42. Cayuela, 2005, p. 125

43. Rueda Ramírez, 2012, p. 70.

44. Herrera Morillas, 2002, p. 44.

45. Moncayo y Gurrea, Rimas, p. 32.

46. Creo que resultaría interesante estudiar la proporción de las coincidencias de los nombres en estas misceláneas junto a su relevancia en la sociedad del tiempo, a su género y nivel social, pero los límites de este trabajo me impiden abarcar ahora este análisis que dejo para una futura investigación. 
Guevara, Antonio Mira de Amescua, Francisco Rojas Zorrilla, Felipe Godínez y Juan Pérez de Montalbán. Como destaca Osuna, Luis Ramírez, «el de la gran memoria» ${ }^{47}$, había logrado reunir en sus Avisos para la muerte además a varios escritores destacados como Juan de Jáuregui, Gabriel Bocángel, Francisco Quintana y José Pellicer. La mayoría de estos autores no solo participó en la Fama póstuma y en las Lágrimas, sino que también -con anterioridad - en varias otras colecciones poéticas colectivas relacionadas con circunstancias festivas ${ }^{48}$, como por ejemplo el Anfiteatro de Felipe el Grande (1631) y los Elogios al Palacio Real del Buen Retiro $(1635)^{49}$. De hecho «los espacios de la socialización poética que propicia la vida ciudadana barroca, y en este caso, de manera más concreta, la vida cortesana» ${ }^{50}$ pueden muy bien darse en ocasiones religiosas o en la despedida fúnebre de un personaje importante ${ }^{51}$. No parece haber la menor duda de que, por debajo de esa apariencia de personales homenajes de funera tan desligados de afanes mundanales, se halle precisamente un tejido de precisas aspiraciones en la Corte que van del simple deseo de entrar en el Parnaso de los poetas españoles, como en el caso de Moreto y Matos Fragoso, hasta el agobio por las envidias que revelan las palabras de Lorenzo de Urnieta i Aguirre en el prólogo a las Lágrimas titulado «Epístola que alaba la virtud a la envidia» (fols. [7r-10v]).

En mi opinión, la proyección social de estas misceláneas nos revela que a la hora de analizar estas obras hay que tener en cuenta tanto la conjunción de lo individual como de lo colectivo que supone la formulación personal del tema por parte de cada autor como su programada reincidencia a lo largo de la colección. Una misma razón llevó a esta plétora de escritores a participar en las misceláneas de funera, es decir, «muchos de ellos buscaban ser recordados como poetas justamente por haber formado parte de la Fama Póstuma» ${ }^{52}$, y de las Lágrimas, añadiría yo, puesto que ningún otro poeta, salvo Lope y Montalbán, gozó de un homenaje de tamaño similar. Retomando las palabras de Juan Antonio de Vera, los poetas cortesanos de la primera mitad del reinado de don Felipe el Grande no solo aspiraban a ser "de Lope" sino que su formación tenía que perfeccionarse hasta ser «addottorato in Lope» (p. 72). Nada mejor que participar en su homenaje in mortem y en el de su estimado discípulo para conseguir laurearse.

47. José de Valdivieso, Aprobación de los Avisos, h. 6r (cit. por Osuna, 2009, p. 47).

48. Ver a este propósito la presencia de Calderón, Montalbán y Villaizán juntos en varias obras colectivas (Cruickshank, 2011, pp. 219-221) antes de que Montalbán y Villaizán se disputasen una comedia (Dixon, 1961).

49. Cruickshank observa sin embargo que Calderón no participó en los Elogios, posiblemente por «haberse visto atado por otros compromisos» (2011, p. 252). Para las posibles razones que llevaron a Calderón a no participar en la Fama póstuma, ver Di Pastena (2001, p. XLVII), y Cruickshank, 2011, pp. 172-173.

50. Osuna, 2009, p. 50

51. Como destaca Wilson, al hablar de la influencia de Ignacio de Loyola en los poemas de los Avisos, «es curioso encontrar juntos en el tomito tantos versos sobre los Novísimos escritos por los poetas cortesanos, tantas veces llamados frívolos por la crítica del siglo XIX» (Wilson, 1972, p. 80).

52. Burguillo, 2019, p. 225. 


\section{BiBLIOGRAFÍA}

Alviti, Roberta, «El proceso de escritura en colaboración: sincronía y diacronía», en La comedia escrita en colaboración en el teatro del Siglo de Oro, ed. Juan Matas Caballero, Valladolid, Ediciones de la Universidad de Valladolid, 2017, pp. 15-27.

Burguillo, Javier, «Un epitafio de Pellicer a la muerte de Lope anterior a la Fama póstuma», Anuario Lope de Vega, 25, 2019, pp. 209-230.

Baranda Leturio, Nieves, «Mujer, escritura y fama: la Hespaña Libertada (1618) de doña Bernarda Ferreira de Lacerda», Península. Revista de estudos ibéricos, 0, 2003, pp. 225-239.

Baranda Leturio, Nieves, «Desterradas del Parnaso. Examen de un monte que solo admitió musas», Bulletin Hispanique, 109-2, 2007, pp. 421-447.

Bresadola, Andrea, «En las honras de Lope Félix de Vega y honores extremos del doctor Juan Pérez de Montalbán: Francisco de Quintana y la oratoria fúnebre del siglo XVII», Lectura y signo, 7, 2012, pp. 67-89.

Cayuela, Anne, Alonso Pérez de Montalbán. Un librero en el Madrid de los Austrias, Madrid, Calambur, 2005.

Cinti, Bruna, «Homenaje a Lope en la Venecia del Seiscientos», Cuadernos Hispanoamericanos, 161-162, 1963, pp. 609-620.

Cinti, Bruna, Letteratura e politica in Juan Antonio de Vera ambasciatore spagnolo a Venezia (1632-1642), Venezia, Libreria Universitaria Editrice, 1966.

Conrieri, Davide, y Vuelta García, Salomé, «Le Essequie poetiche per Lope de Vega: bilancio e prospettive», en Forme e occasioni dell'encomio tra Cinque e Seicento, ed. Danielle Boillet y Liliana Grassi, Lucca, Maria Pacini Fazzi Editore, 2011, pp. 313-343.

Cruickshank, Don W., Calderón de la Barca. Su carrera secular, Madrid, Gredos, 2011.

Dadson, Trevor J., «La difusión de la poesía española impresa en el siglo XVII», BuIletin hispanique, 113.1, 2011, pp. 13-42.

Del Piero, Raúl A., «La respuesta de Pérez de Montalbán a la Perinola de Quevedo», PMLA, 76.1, 1961, pp. 40-47.

De l'ambassadeur: Les écrits relatifs à l'ambassadeur et à l'art de négocier du Moyen Âge au début du XIxe siècle, ed. Stefano Andreatta, Stéphane Péquinot y Jean-Claude Waquet, Rome, Publications de l'École française de Rome, 2015. Disponible en línea: http://books.openedition.org/efr/2887. 
Demattè, Claudia, «Mélanges et littérature mêlée: de la Dorotea de Lope de Vega (1632) à le Para todos de Juan Pérez de Montalbán (1632)», en Ouvrages miscellaneées et théories de la connaissance à la Renaissance, ed. Dominique de Courcelles, Paris, École Nationale des Chartes, 2003, pp. 185-195.

Demattè, Claudia, «Entre ingenios anda el juego: Juan Pérez de Montalbán y las comedias en colaboración con Lope y Calderón. Notas acerca de Los terceros de san Francisco», en La comedia escrita en colaboración en el teatro del Siglo de Oro, ed. Juan Matas Caballero, Valladolid, Ediciones de la Universidad de Valladolid, 2017, pp. 229-242.

Demattè, Claudia, «Estudio preliminar», en Juan Pérez de Montalbán, Segundo tomo de comedias, vol. 2.1, Kassel, Reichenberger, 2019, pp. 3-11.

Di Pastena, Enrico, «Introducción», en Fama póstuma a la vida y muerte del doctor frey Lope Félix de Vega Carpio y elogios panegíricos a la inmortalidad de su nombre, ed. Pérez de Montalbán, Juan, Pisa, Edizioni ETS, 2001, pp. I-CXII.

Dixon, Victor, «Apuntes sobre la vida y obra de Jerónimo de Villaizán y Garcés», Hispanófila, 13, 1961, pp. 5-22.

Dixon, Victor, «New (and Ancient) Lights on the Life of Juan Pérez de Montalbán», Bulletin of Spanish Studies, 90, 4-5, 2013, pp. 509-534.

Entrambasaguas y Peña, Joaquín de, «Elegía de Lope de Vega en la muerte de Jerónimo de Villaizán», Fénix. Revista del Tricentenario de Lope de Vega, 1, 1935, pp. 127-144.

Essequie poetiche ovvero lamento delle muse italiane in morte del signor Lope de Vega insigne ed incomparabile poeta spagnuolo, en Obras sueltas de Lope de Vega, vol. XXI, Madrid, Antonio de Sancha, 1779, pp. 1-165.

Errico, Scipione, Le rivolte del Parnaso. Commedia in cinque atti, ed. G. Santangelo, Catania, Società di Storia patria per la Sicilia Orientale, 1974.

Fernández-Daza Álvarez, Carmen, «Lope de Vega y Juan Antonio de Vera», Anuario de estudios filológicos, 17, 1994, pp. 115-131.

García-Reidy, Alejandro, «Dinámicas y retóricas de la sociabilidad literaria en los volúmenes-homenaje al escritor fallecido», en Ámbitos artísticos y literarios de sociabilidad en los Siglos de Oro, ed. Elena Martínez Carro y Alejandra Ulla Lorenzo, Kassel, Reichenberger, 2020, pp. 217-223.

Hempel, Wido, In onor delle fenice ibera: esequie di Lope de Vega, Über die «Essequie poetiche di Lope de Vega», Venedig 1636, nebst einer kommentierten ausgabe der "Orazione del Cavallier Marino» und des «Ragguaglio di Parnaso», Frankfurt am Main, Klostermann, 1964.

Herrera Morillas, José Luis, «Libros impresos en Sevilla durante los siglos XV al XVIII, conservados en las bibliotecas universitarias de Andalucía», Boletín de la Asociación Andaluza de Bibliotecarios, 68.9, 2002, pp. 37-66. 
Kennedy, Ruth Lee, «The Close Friendship of Gabriel Téllez and Pérez de Montalván: its Possible Significance for the Segunda parte of Tirso», Revista Hispánica Moderna, 37.4, 1972-1973, pp. 262-282.

Kennedy, Ruth Lee, «Addenda to "The Close Friendship of Gabriel Téllez and Pérez de Montalván: its Possible Significance for the Segunda parte of Tirso"», Revista Hispánica Moderna, 38.3, 1973-1974, pp. 78-84.

Lágrimas panegíricas a la temprana muerte del gran poeta y teólogo insigne doctor Juan Pérez de Montalbán, clérigo presbítero y Notario de la Santa Inquisición, natural de la imperial villa de Madrid, lloradas y vertidas por los más ilustres ingenios de España, ed. Pedro Grande de Tena, Madrid, Alonso Pérez, 1639. Biblioteca de Catalunya: R(8)-8-329.

López-Cordón Cortezo, María Victoria, «Juan Antonio de Vera y Zúñiga (1583-1658). Modello di ambasciatori o specchio di trattatisti?», en De l'ambassadeur: Les écrits relatifs à l'ambassadeur et à l'art de négocier du Moyen Âge au début du XIxe siècle, ed. Stefano Andreatta, Stéphane Péquinot y Jean-Claude Waquet, Rome, Publications de l'École française de Rome, 2015, pp. 337-361. Disponible en línea: http://books.openedition.org/efr/2887 (fecha de consulta: 20/08/2019).

Luna, Lola, Leyendo como una mujer la imagen de la mujer, Barcelona, Anthropos, 1996.

Maggi, Marco, «"Sconcertati concerti": Tesauro, la Supposition e l'Arte nuevo», Lettere italiane, 55.3, 2003, pp. 417-442.

Marcello, Elena, «Lope de Vega en Italia. De dramaturgo a personaje de ficción», en Pictavia Aurea, Anejos de Criticón, 19, 2013, pp. 955-962.

Martos Pérez, María Dolores, «De musas a poetas: escritoras y enunciación canonizadora en la obra de Lope de Vega», Arte nuevo, 4, 2017, pp. 787-847.

Merluzzi, Manfredi, «Juan de Vera e I'Italia. Dall'ispirazione letteraria alla pratica diplomatica», en De l'ambassadeur: Les écrits relatifs à l'ambassadeur et à l'art de négocier du Moyen Âge au début du xıxe siècle, ed. Stefano Andreatta, Stéphane Péquinot y Jean-Claude Waquet, Rome, Publications de l'École française de Rome, 2015, pp. 363-377. Disponible en línea: http://books.openedition.org/efr/2887 (fecha de consulta: 20/08/2019).

Moncayo y Gurrea, Juan de, Rimas, Zaragoza, Diego Dormer, 1652.

Osuna, Inmaculada, «Los Avisos para la muerte de Luis Ramírez de Arellano», Via Spiritus, 16, 2009, pp. 45-82.

Pérez de Montalbán, Juan (ed.), Fama póstuma a la vida y muerte del doctor frey Lope Félix de Vega Carpio y elogios panegíricos a la inmortalidad de su nombre, ed. Enrico Di Pastena, Pisa, Edizioni ETS, 2001.

Pérez de Montalbán, Juan, Obras no dramáticas, ed. Enrique Laplana Gil, Madrid, Gredos, 1999. 
Pérez de Montalbán, Juan, Primer tomo de comedias, ed. Claudia Demattè, vol. 1.4: El mariscal de Virón, ed. María Moya García; La toquera vizcaína, ed. Enrico Di Pastena; Amor, privanza y castigo, ed. Josefa Badía Herrera, Kassel, Reichenberger, 2018.

Pineda, Victoria, «La elocuencia del embajador», Studia Aurea, 9, 2015, pp. 483-530.

Plata, Fernando, «La polémica en torno a La Perinola de Quevedo con un texto inédito», La Perinola. Revista de investigación quevediana, 10, 2006, pp. 245-255.

Profeti, Maria Grazia, «ll Pellicer e la sua Idea de la comedia de Castilla, deducida de las obras cómicas del Doctor Juan Pérez de Montalbán», en Miscellanea di studi ispanici, Pisa, Università di Pisa, 1966-1967, pp. 170-217.

Profeti, Maria Grazia, «El claustro y la pluma: Lope de Vega y la mujer culta», InTRAlinea, Special Issue, Palabras con aroma a mujer. Scritti in onore di Alessandra Melloni, ed. María Isabel Fernández García y Mariachiara Russo, 2005, s. p. Disponible en línea: http://www.intralinea.org/specials/article/2005 (fecha de consulta: 10/07/2019).

Rojas Zorrilla, Francisco, Obras completas. Volumen VI: Segunda parte de comedias, ed. Rafael González Cañal, Felipe Pedraza Jiménez y Milagros Rodríguez Cáceres, Cuenca, Ediciones de la Universidad de Castilla-La Mancha, 2018.

Rueda Ramírez, Pedro, «Los libreros Juan López Román y Antonio de Toro en la carrera de Indias», en El libro en circulación en el mundo moderno en España y en América, ed. Pedro Rueda Ramírez, Madrid, Calambur, 2012, pp. 46-71.

Tedesco, Anna, «"Scrivere a gusti del popolo": I'Arte nuevo di Lope de Vega nell'Italia del Seicento», II Saggiatore musicale, 13.2, 2006, pp. 221-245.

Trambaioli, Marcella, «La fama póstuma de Lope de Vega», Studia Aurea, 10, 2016, pp. 173-199.

Sánchez Jiménez, Antonio, «La apreciación de la obra de Lope de Vega entre la Fama póstuma (1636) y el Diccionario de Autoridades (1726-1737)», Anuario Lope de Vega, 17, 2011, pp. 123-149.

Vega García-Luengos, Germán, «Cómo Calderón desplazó a Lope de los aposentos: un episodio temprano de ediciones espurias», en Calderón: innovación y legado, ed. Ignacio Arellano y German Vega García-Luengos, New York, Peter Lang, 2001, pp. 367-384.

Vega y Carpio, Lope de, Colección de las obras sueltas, así en prosa como en verso, tomo XXI, Madrid, Antonio de Sancha, 1779. 
Vuelta García, Salomé, «Lope de Vega nel Parnaso italiano del Seicento», en «Por tal variedad tiene belleza». Omaggio a Maria Grazia Profeti, ed. Antonella Gallo y Katerina Vaiopoulos, Firenze, Alinea, 2012, pp. 271-280.

Wilson, Edward M., «Un romance ascético de Calderón: "Agora, Señor, agora..."», Boletín de la Real Academia Española, 52, 195, 1972, pp. 79-105.

Zimic, Stanislav, «Francisco de Quintana, un novelista olvidado, amigo de Lope de Vega», Boletín de la Biblioteca Menéndez Pelayo, 51, 1975, pp. 169-232. 